\title{
pro.posıções
}

DOSSIÊ: "Vertentes da educação inclusiva".

\section{Caminos y prácticas evaluativas en la educación especial: experiencias en Brasil y Argentina. ${ }^{1}$}

\section{Paths and evaluation practices in special education: experiences in Brazil and Argentina}

\author{
Morgana de Fátima Agostini Martins (i) \\ Carina Viviana Kaplan (ii) \\ Marta Liliana Sipes (iii)
}

\begin{abstract}
(i) Universidade Federal da Grande Dourados; Dourados, MS, Brasil, http://orcid.org/0000-00019117-1320,morganamartins@ufgd.edu.br

(ii) Universidad de Buenos Aires, Buenos Aires; Universidad Nacional de La Plata, Buenos Aires. Argentina. kaplancarina@gmail.com

(iii) Universidad de Buenos Aires, Buenos Aires; Argentina. Universidad Del Salvador. Buenos Aires. Argentina.martasipes@gmail.com
\end{abstract}

Resumen: Los procesos evaluativos que se llevan adelante con la finalidad de definir los soportes y los apoyos pedagógicos a los alumnos con discapacidades han sido tema de diversos estudios en Brasil y Argentina. Los profesores y los maestros, debido a su contacto directo con los alumnos, son los profesionales más indicados para realizar una evaluación diagnóstica de los apoyos pedagógicos necesarios en los casos de dificultades de aprendizaje. En este artículo, se establece un análisis de los procesos evaluativos y de las modalidades de diagnóstico reconocidos y aceptados en la Ciudad Autónoma de Buenos Aires, Argentina, y en la ciudad de Dourados, Mato Grosso do Sul, Brasil. La propuesta es recorrer las prácticas evaluativas que se utilizan en esos procesos para la identificación y la derivación de niños con discapacidades a los centros de apoyo escolar o a los servicios de asistencia existentes en cada una de las dos ciudades.

Palabras clave: educación especial, evaluación, apoyos pedagógicos específicos, inclusión escolar

\footnotetext{
1 Apoyo: Coordenação de Aperfeiçoamento de Pessoal de Nível Superior (CAPES)
} 
Abstract: The evaluation procedures that set the supports for students with disabilities have been the subject of many studies in Brazil and Argentina. The teachers, because they are in direct contact with the students, are the professionals best suited to do a diagnostic evaluation of the support needed, when difficulties are detected. This article analyzes the diagnostic procedures recognized and accepted in the province of Buenos Aires, Argentina, and in the municipality of Dourados, Mato Grosso do Sul, Brazil. This research observes the evaluation practices for the identification of children with disabilities, and for the referral of these children to the support services in the two municipalities.

Keywords: special education, evaluation, specific support, school inclusion

\section{Políticas de inclusión}

Actualmente existen debates académicos y gran cantidad de papers respecto de las transformaciones de los sistemas educativos, las cuales partieron de la generalización -es decir, de la globalización- de las políticas de inclusión. En ese contexto, son centrales, específicamente, los procesos de integración escolar, que impulsan la escolarización en escuelas comunes de los alumnos con restricciones o con las denominadas discapacidades. ${ }^{2}$ Esos debates comprenden aspectos epistemológicos, ideológicos, pedagógicos y didácticos acerca de la naturaleza del campo de estudio en que se compromete la toma de decisiones, y modalidades de intervención psicoeducativas sobre las necesidades de alumnos, familias y escuelas. Lo que lo convierte en un ramo dilemático y dinámico.

Las actuales políticas educativas inclusivas de carácter regional han otorgado visibilidad y prioridad al "campo" de los alumnos con discapacidades (restricciones) y/o con dificultades de aprendizaje, lo que ha ampliado el análisis y el estudio de los aspectos de los currículos escolares, en los cuales hubo avances extraordinarios. Desde los currículos específicos para cada restricción ${ }^{3}$ o para superdotación, el aspecto curricular, tanto en Brasil como en Argentina, pasó por estos períodos o etapas, que -como capas geológicas- coexisten en el presente: el incomprendido concepto de "adecuaciones curriculares", la exclusión de algunos alumnos de sistemas escolares "por no alcanzar los logros curriculares" y las actuales ideas de

\footnotetext{
2 Subrayamos que esos estudiantes, hasta hace muy poco tiempo, solo podían cursar sus estudios en las escuelas de educación especial, según la especificidad de su restricción.

${ }^{3}$ Diseños curriculares para alumnos ciegos y para estudiantes con deficiencias mentales, con sordera o con severos trastornos de la personalidad/trastorno del espectro del autismo.
} 


\section{pro.posıções \\ $e$-ISSN 1980-6248}

http://dx.doi.org/10.1590/1980-6248-2017-0116

que haya un currículo único, o sea, un mismo marco curricular para todos los alumnos del sistema.

El camino recorrido fue una vía de luchas y controversias, de "visibilización" de los derechos de infancias que, durante siglos, estuvieron segregadas. Sin embargo, los logros no se han alcanzado de una vez y para siempre ni en la dimensión teórica ni en la dimensión de las prácticas escolares cotidianas.

Advertimos que los movimientos pendulares son frecuentes en la temática que investigamos, porque existen vectores de tensiones que atraviesan el campo y las discusiones. Indudablemente, hay comprometidos aspectos ideológicos, las relaciones de fuerza, la concepción de calidad educativa, el alcance de la escolarización, las diferencias y la igualdad, entre otros. Cuando se discuten aspectos curriculares, no solo se discute el currículo escolar.

\section{Evaluación diagnóstica}

Dentro de esas discusiones, debates y controversias, la evaluación diagnóstica cobra una importancia sustancial. La evaluación diagnóstica es la que se supone que guía las acciones de los agentes psicoeducativos y de las instituciones escolares. Existe la creencia que determinadas estrategias evaluativas - por ejemplo, las psicométricas (las mediciones de la llamada inteligencia) - darán pistas sobre lo "que hay que hacer" en la escuela. Esa concepción —errada - se muestra como privilegiada por sobre las estrategias pedagógico-didácticas específicas de la escuela.

El uso de las pruebas psicométricas en el contexto escolar puede arrojar resultados ineficaces. En la actualidad, ya no pueden considerarse dichas pruebas (tests) como neutrales u objetivas; ellas están desligadas del contexto y de los sujetos que las administran y a quienes se les administran. El uso frecuente de esos tests nos ha hecho comprender que esa estrategia promueve hipótesis diagnósticas evaluativas que poco colaboran con las preocupaciones de la escuela y, sobre todo, del maestro, que es quien tiene que elaborar estrategias pedagógicas adecuadas a cada uno de sus alumnos (Sipes, 2012). Además de eso, debemos reconocer que los resultados obtenidos por medio de esos tests no le ayudan al profesor a crear modalidades de enseñanza, tampoco le auxilian a seleccionar contenidos y a elaborar currículos. 


\section{pro.posıções}

http://dx.doi.org/10.1590/1980-6248-2017-0116

\section{$e$-ISSN 1980-6248}

Coincidiendo con lo que plantea Valsiner (2012), "el imperativo de convertir cualquier fenómeno en datos mediante la cuantificación -de 'adjuntar números' [comillas en el original] al problema original- ha conducido al extravío de la disciplina durante alrededor de un siglo" (p.139). El desvío, podríamos agregar, trasunta la disciplina psicológica, o la psicopedagógica, y se encapsula en los hechos cotidianos de la escuela. No son las técnicas psicométricas las que juegan el papel principal; las técnicas psicométricas protocolizadas guardan relación con las modalidades que asumen las prácticas pedagógicas, evaluativas y psicoeducativas dentro del marco político. De allí se hace necesario situar al sujeto a evaluar dentro de un marco referencial inescindible y considerar el pasaje por la escuela como un "vector" del desarrollo (Rivière, 2003).

\section{Posibilidades de apoyo}

Entre las evaluaciones diagnósticas protocolizadas y el conocimiento de aquello que el alumno realmente necesita - como apoyos específicos- para poder aprender, hay una gran distancia y muchas diferencias. Las pruebas psicométricas de apoyo a la escolarización son suministradas por profesionales de la salud, quienes luego transfieren los resultados a los maestros a cargo de los alumnos. Esos datos, así aportados, no logran ser suficientes para encarar y dirigir la tarea áulica. Del mismo modo, los diagnósticos de las llamadas patologías o síndromes no dan cuenta de las posibilidades y de las peculiaridades de aprendizaje. Es por esa razón que quienes devienen artífices del plan de acción del cotidiano del aula son los propios docentes, que pueden indicar cuáles son las posibilidades de apoyo necesarias para trabajar pedagógicamente con cada alumno.

Para ello, en el tiempo actual, en la Ciudad Autónoma de Buenos Aires (CABA), las escuelas están utilizando un Plan Pedagógico Individual que contiene los siguientes ítems:

- Informe de situación de aprendizaje. Contexto áulico; contexto institucional; contexto familiar; contexto comunitario.

- Estrategias de integración: Horarios; concurrencia de otros profesionales.

- Propuesta curricular individualizada. 


\section{pro.posıções}

http://dx.doi.org/10.1590/1980-6248-2017-0116

\section{$e$-ISSN 1980-6248}

- Posibilidades de apoyo.

Esa manera de pensar las evaluaciones y los procesos de integración escolar dan cuenta de una transformación importante en las escuelas y en las concepciones de los agentes que la habitan. Es por esa razón que resulta indispensable historizar esos procesos para entender cuál es la génesis de sus tensiones.

Del mismo modo, en la ciudad de Dourados, los profesores especializados han construido un Plan Educativo Individualizado articulado con las necesidades del alumno. Ese plan ha sido la "guía" del trabajo ofrecido como Atención Educativa Especializada (AEE), que es el servicio principal dirigido a los niños y jóvenes con restricciones o discapacidades, y obedece a un modelo único de atención a ellos. La Atención Educativa Especializada se lleva a cabo en las Salas de Recursos Multifuncionales (SRM), instauradas en escuelas de todo el país desde 2008. Ese servicio ha sido ponderado por la Política Nacional de Educación Especial desde la Perspectiva de Educación Inclusiva (Brasil, 2008). La comprensión de la construcción de ese modelo demanda un recorrido histórico de la Educación Especial en Brasil.

En la década de 1960, movimientos sociales empezaron a preocuparse por la inclusión de una parte de la población en el ambiente escolar. Mendes (2006) afirma que la inclusión de todos en la escuela no atiende únicamente a la necesidad de escolarizarlos, sino que forma parte de una lucha cuyos objetivos son garantizar los derechos de los individuos considerados "diferentes" y combatir la segregación educativa, justificada por la creencia de que las necesidades de eses sujetos podrían ser satisfechas si estos recibieran enseñanza en ambientes separados (escuelas de educación especial). Hoy, en vista de la inclusión escolar de todos independientemente de la clase social, del color, de la etnia y de las necesidades educativasesa creencia parece ser un ideal superado.

Durante mucho tiempo, los alumnos con alguna necesidad educativa especial fueron considerados incapaces de aprender e integrarse en la sociedad. De ese modo, eran separados de los otros, estudiaban en escuelas o salas especiales y no tenían posibilidades de ingresar en escuelas comunes. Más tarde, debido al aumento significativo de profesionales y, sobre todo, a la lucha de los padres por derechos y mejores y adecuadas condiciones de enseñanza para esos alumnos, se inició la búsqueda de garantías del cumplimiento de las leyes que priorizan los 


\section{pro.posições \\ $e$-ISSN 1980-6248}

http://dx.doi.org/10.1590/1980-6248-2017-0116

principios de los Derechos Humanos, como el derecho a la educación y a la atención educativa en sus ambientes naturales, con personas de su franja etaria.

A partir de la década de 1990, las declaraciones internacionales de Salamanca (Unesco, 1994) reforzaron la creación y la promulgación de leyes que pudieran asegurarles a esos "nuevos estudiantes" el ingreso y la permanencia en las escuelas comunes. En Brasil, la inclusión escolar y el respeto a las habilidades y a las necesidades de aprendizaje de esos alumnos están asegurados desde la Constitución Federal de 1988.

Entre las varias medidas tomadas para garantizar la inclusión escolar de los alumnos que se encuadran en el Público Objetivo de la Educación Especial (POEE), o sea, que poseen certificado médico de discapacidades, trastornos generalizados del desarrollo y altas habilidades/superdotación (Brasil, 2010), la principal ha sido la creación de Salas de Recursos Multifuncionales (SRM). Según una Nota Técnica del Ministerio de Educación brasileño, las SRM organizan espacios en la propia escuela común, los cuáles son dotados de equipos, recursos de accesibilidad y materiales pedagógicos que ayudan a promover la escolarización y eliminan las barreras que impiden la plena participación de los alumnos que son el público objetivo de la educación especial, con autonomía e independencia, en el ámbito educativo y social (Brasil, 2010).

El Decreto n. 7.611/11 (Decreto BR, 2011) define las Salas de Recursos Multifuncionales como ambientes dotados de equipos, muebles y materiales didácticos y pedagógicos para el ofrecimiento de la AEE. Conforme el decreto, esos espacios deben: I) proveer condiciones de acceso, participación y aprendizaje en la educación común y garantizar servicios de apoyo especializados y adecuados a las necesidades individuales de los estudiantes; II) asegurar la transversalidad en las acciones de la educación especial en la educación común; III) fomentar el desarrollo de recursos didácticos y pedagógicos que eliminen las barreras del proceso de enseñanza y aprendizaje; IV) proporcionar condiciones para la continuidad de los estudios en todos los niveles, las etapas y las modalidades de enseñanza.

Cabe señalar que la lucha por la inclusión escolar y la implementación o creación de nuevos medios de enseñanza es una búsqueda constante, que todavía exige la generación de nuevos caminos. Frente a las posibilidades de discusión respecto de ese servicio (Mendes, Cia, 


\section{pro.posıções}

$e$-ISSN 1980-6248

\& D’Affonseca, 2015), nos concentraremos en las prácticas evaluativas realizadas en las SRM para identificar a los alumnos "elegibles" para la AEE.

\section{Análisis de algunos procesos evaluativos}

Para que el alumno pueda ingresar en ese servicio, hay que ser evaluado por un profesor especializado en educación especial. A pesar de eso, en muchos casos, la presentación de certificado médico es el único requisito (Milanesi, 2012; Veltrone \& Mendes, 2011).

Trabajos realizados en el marco del Grupo de Investigaciones en Educación Especial (GEPES), en colaboración con el estudio en red del Observatorio Nacional de Educación Especial (ONEESP), con el objetivo de analizar las "Salas de Recursos Multifuncionales" en Brasil, detectaron errores y omisiones en esas evaluaciones tomadas en todo el territorio nacional. Además, los veinte y cinco investigadores, provenientes de dieciséis estados brasileños, que llevaron a cabo esos estudios subrayaron que la preparación de los profesores para suministrarlas era escasa o nula (Mendes et al., 2015; Milanesi, 2012).

En Dourados, los trabajos de Anache, Silva, Bruno y Martins (2015); Melo (2014); Melo y Martins (2013) investigaron y discutieron las prácticas evaluativas de los profesores de las SRM. Los investigadores constataron que las evaluaciones aún están sustentadas desde el modelo médico — con énfasis en las carencias del alumno, en sus inhabilidades e incapacidades- y destacan el valor soberano de los certificados médicos y psicológicos -basados en el análisis genético y en el cociente intelectual- en la práctica pedagógica del docente. Los procesos evaluativos propuestos en las SRM constituyen evaluaciones de derivación (para el diagnóstico o para la identificación), prueba dirigida para la construcción del plan educativo individualizado y examen de rendimiento escolar.

Según Anache, Rovetto y Oliveira (2014), la evaluación en el contexto de la educación especial envuelve los criterios de identificación de su público y es realizada prioritariamente en los ámbitos de la salud y de la educación. Es una relación compleja, pues la atención educativa especializada considera el certificado médico en la planificación de los apoyos necesarios para proveer a los alumnos identificados posibilidades educativas en el sistema de educación común. 


\section{pro.posıções}

http://dx.doi.org/10.1590/1980-6248-2017-0116

\section{$e$-ISSN 1980-6248}

Muchas veces, el alumno es encaminado al servicio ofrecido en las SRM cuando el adulto responsable del niño presenta su certificado médico. En ese momento, tal documento se vuelve fundamental para garantizar su vacante.

Así como en Brasil, en la Ciudad Autónoma de Buenos Aires, los procesos diagnósticos les posibilitan a los investigadores una gran cantidad de dimensiones de análisis. Señalamos algunos aspectos importantes:

- Las estrategias diagnósticas; los instrumentos o pruebas (tests) utilizados; así como las categorías donde se encuadran los resultados de las pruebas.

- La ilusión de asepsia de los procesos —en algunos casos se suministra toda la prueba tan como está prescrita, en otras se suministra tan sólo un fragmento del test.

- Las concepciones acerca de las restricciones cognitivas de los sujetos —si son permanentes, momentáneas, innatas, adquiridas, coyunturales, estructurales, si pueden variar con adecuadas interacciones pedagógicas.

- Los diagnósticos, casi en su totalidad, tienden a poner de relieve lo que "al alumno le falta", sin hacer mención al tipo de estrategia pedagógica necesaria.

Se produce en estos procesos diagnósticos una suerte de discontinuidad o desacople entre los resultados de los tests y las posibilidades de que ese dato se transforme en estrategias pedagógicas adecuadas a ese sujeto.

Existe un tipo de evaluación llamada diagnóstica o de identificación, que permite escuchar la apreciación del docente. En el caso de los alumnos que no poseen certificado médico y presentan alguna dificultad durante su vida académica (en el proceso de aprendizaje, en la conducta, entre otros), los profesores de la sala de clase común los conducen a una evaluación suministrada por un docente especializado. Es la llamada etapa de sondeo.

De acuerdo con Fernandes y Viana (2009), los profesores, debido a su contacto directo con los alumnos en sala de clase, son los profesionales de la educación más indicados para realizar una evaluación diagnóstica. Pueden observar formal e informalmente las actividades y las conductas reveladas en el cotidiano y señalar las capacidades generales y específicas. Es la evaluación diagnóstica la que determina si los estudiantes tienen o no alguna 


\section{pro.posıções}

http://dx.doi.org/10.1590/1980-6248-2017-0116

\section{$e$-ISSN 1980-6248}

Necesidad Educativa Especial (NEE); y, basados en los resultados, los docentes definen la potencial "elegibilidad" de los alumnos para (derivación hacia) la atención especializada.

Anache et al. (2015), en investigación llevada a cabo en las SRM de tres ciudades del estado de Mato Grosso del Sul, constataron que los profesores utilizan su propia trayectoria profesional y algunos pocos conocimientos adquiridos a lo largo del trabajo en la educación especial para identificar y planificar futuras intervenciones en la Atención Educativa Especializada (AEE), a pesar de que los docentes no poseen formación específica para eso. Entonces, el profesor es el agente principal de la evaluación pedagógica, pero, muchas veces, deviene un colaborador de evaluaciones clínicas y de formulación de hipótesis diagnósticas, sometido a aquello que menciona y determina el certificado médico.

En Argentina, las derivaciones de educación común a educación especial fueron identificadas en dos procesos diferentes. El primero está vinculado a la derivación de alumnos de escuelas de educación común a escuelas de educación especial. El segundo tiene que ver con los pedidos de intervención que se realizan de las escuelas de educación común a los equipos de apoyo a la escolaridad para que intervengan a través de una maestra de "apoyo a la inclusión" para realizar apoyos pedagógicos en la escolaridad de niños que cursan sus estudios en la escuela común en carácter de "integrados" e "incluidos", y son considerados, en la matrícula, como estudiantes de educación especial.

Este campo de estudio dilemático, que intenta encontrar un diagnóstico, generalmente tiene a los alumnos como destinatarios patológicos de una mitología predefinida e inflexible. Intenta articular las peculiaridades del sujeto con el aprendizaje escolar.

El problema de la explicación del desarrollo y del aprendizaje, o, si se quiere, de la explicación psicológica, se ha planteado, a su vez, con respecto a reconocer los límites de un intento explicativo causal-lineal (Baquero, 2009; Castorina, 2007). Tras las explicaciones médicas y psicológicas, los niños con discapacidad o restricciones cognitivas suelen ser excluidos, estigmatizados y etiquetados por su condición como alguien menor, deficitario, incompleto. Tales rótulos resultan de un proceso evaluativo reducido; desarrollado por un especialista clínico que, la mayoría de las veces, no conoce el cotidiano escolar.

En Brasil, durante mucho tiempo y, en Argentina, todavía hoy, ese rótulo permitió/permite la derivación de niños a espacios segregados de enseñanza, sobre todo, a 


\section{pro.posıções \\ $e$-ISSN 1980-6248}

http://dx.doi.org/10.1590/1980-6248-2017-0116

escuelas de educación especial. Actualmente, en los dos países, la práctica de derivación a escuelas de educación especial ha disminuido drásticamente, bajo la égida de la inclusión escolar.

En el caso de Brasil, esa práctica ha sido disimulada por medio de las llamadas Salas de Recursos Multifuncionales (Martins \& Sipes, 2015). Aunque el alumno esté en la escuela común y, teóricamente, conviva con los niños con desarrollo típico, la derivación a la atención educativa en esas salas crea la imagen de diferente, problemático y discapacitado. Uno de los indicios es que, si bien la atención educativa especializada deba ocurrir a contraturno, como determina la ley, ella, de hecho, ocurre cuando el niño debería estar con los demás alumnos, en su sala de clase. Entonces, la derivación de los niños a las SRM, además de perjudicar su rendimiento escolar, fortalece la exclusión.

Ese niño todavía es entendido como alguien que debe adecuarse, porque los déficits son solo suyos. En ese contexto, la necesidad de apoyo por parte del alumno lo vuelve aún menos importante e incompetente en sus relaciones con los demás.

Según Elias y Scotson (2000), la autoimagen y la autoestima de un individuo están vinculadas con lo que los otros miembros del grupo piensan de él. El estigma impuesto por el grupo de establecidos a los outsiders tiende a formar parte de su autoimagen, lo que les debilita a los individuos de ese segundo grupo y los mantiene en este. El acceso desigual a las fuentes de poder en la figuración escolar alimenta esa condición. De acuerdo con sus especificidades, cada alumno debe recibir diferentes atenciones educativas, sin que eso constituya un demérito o favorezca el desencadenamiento de un proceso de marginación, ya que todas las personas son diferentes y deberían poder convivir a partir de esa diferenciación (Schneider \& Ravasio, 2014).

Recurriendo a Elias (1994), sostenemos que los individuos, en una sociedad, están unidos unos a los otros en redes que no pueden distinguirse a simple vista. Tales redes pueden concebirse como figuraciones y configuraciones posibles en las relaciones entre los sujetos. El repertorio completo de patrones sociales de autorregulación que la persona debe desarrollar internamente, al crecer y devenir un individuo único, es específico de cada generación y, por tanto, en un sentido más amplio, específico de cada sociedad (Elias, 1994). 


\section{pro.posıções}

http://dx.doi.org/10.1590/1980-6248-2017-0116

\section{$e$-ISSN 1980-6248}

Si los seres humanos no fueran, por naturaleza, mucho más maleables y móviles que los animales en cuanto al control conductual, no compondrían juntos un continuum histórico autónomo (una sociedad), tampoco poseerían individualidad propia (Elias, 1994). La capacidad de cambio y de adaptación de los seres humanos es muy grande. Por eso, se debe invertir en la formación de los profesores, comprender la importancia de ella y, aún más, proponer discusiones acerca de la necesidad de ampliar las prácticas que promuevan la inclusión escolar, principalmente en lo que respecta a las prácticas evaluativas. Ese movimiento puede favorecer la inclusión de los niños en escuelas que sean menos excluyentes y tengan acciones más eficaces sobre su desarrollo.

Solidarias de las concepciones que constituyeron las posiciones dominantes en nuestros campos, las explicaciones utilizadas en las derivaciones operaron, en general, una reducción de la complejidad de los problemas a explicar; por otra parte, tendían, por lo mismo, a perder la especificidad de las características del aprendizaje escolar. Puede remitirse una parte sustantiva del problema a la filosofía de la escisión, que dominó buena porción del pensamiento moderno (Castorina, 2007), escisión entre naturaleza y cultura, lo individual y lo social, lo interno y lo externo, etc.

Cuando son matriculados en escuelas comunes, los niños con discapacidad no siempre son, inmediatamente, así identificados. En la ciudad de Dourados/MS, se ha verificado que, durante la matrícula o inscripción, el adulto responsable del niño no informa sus necesidades y/o su discapacidad. Eso se debe al temor que tienen los padres del prejuicio y del rechazo por parte de la escuela o, en muchos casos, al desconocimiento de las restricciones de su hijo. También en las escuelas comunes de la CABA, muchas veces, los responsables de los niños se rehúsan a presentar los documentos solicitados en el caso.

Resulta interesante que las escuelas tengan esas informaciones ya en la matrícula, período de inscripción, pues eso puede ayudarle al profesor a elaborar estrategias y capacitarse en todo lo que sea necesario para recibir y enseñar a ese alumno. Lo que muchas veces no ocurre, porque los padres desconocen una posible discapacidad del hijo, no saben qué le pasará al niño o tienen miedo a que le nieguen la matrícula o lo rechacen.

En el caso del niño que llega a la escuela ya con un informe médico, diagnosticado con algún tipo de discapacidad, no hay evaluaciones para la derivación, y el alumno es 


\section{pro.posıções}

$e$-ISSN 1980-6248

http://dx.doi.org/10.1590/1980-6248-2017-0116

inmediatamente matriculado en la SRM. Tal procedimiento nos remite a la estigmatización, tal como ocurría desde hace más de treinta años. No existe una propuesta de evaluación preliminar, de carácter pedagógico y funcional, que contribuya al reconocimiento de la necesidad de Atención Educativa Especializada y que determine, aún más, si la AEE beneficiará al niño. No hay evaluación de las competencias ni de las necesidades. La etiqueta clínica es lo suficiente para que el niño sea "derivado" sin previsión de cuándo dejará de recibir Atención Educativa Especializada.

La evaluación para la derivación es suministrada por profesores sin esa calificación, lo que produce un inconveniente en los procesos de enseñanza y aprendizaje del alumno, que permanece en las salas de recursos mucho más tiempo que lo necesario. Por otra parte, en los casos en los que el alumno llega ya con el certificado médico puede que el diagnóstico sea precipitado y que el profesor, por no tener autonomía, planifique su trabajo a partir del diagnóstico hecho por otro profesional, que, la mayoría de las veces, no tiene contacto con los profesores, ni con la escuela.

No hay previsión de "alta". Sin objetivos claros en cuanto al desarrollo y al aprendizaje del niño, es imposible prever una fecha de suspensión o finalización de las ayudas y de los apoyos. De acuerdo con Veltrone (2011), hoy, tenemos la perspectiva de agregar también una evaluación del aprendizaje de este alumno que, más que indicar la discapacidad, busque identificar sus necesidades educativas y los caminos necesarios para el éxito en sus trayectorias escolares.

La importancia de las recientes investigaciones reside en que la comprensión de los procesos evaluativos promueve los cambios necesarios. Como complemento de esa afirmación, algunas investigaciones realizadas en Brasil, organizadas por Mendes et al., (2015) han expuesto que no existe un documento que guíe a los profesores en la realización de las evaluaciones pedagógicas y que defina la derivación.

\section{Conclusión}

Conociendo a los peligros de la rotulación, o del etiquetamiento (Kaplan, 2008), basada en un diagnóstico médico, en el contexto educativo, debemos estar atentos a los 


\section{pro.posıções}

http://dx.doi.org/10.1590/1980-6248-2017-0116

\section{$e$-ISSN 1980-6248}

procesos evaluativos y a las derivaciones de niños. Precisamos pensar en estrategias que los evalúen con la intención de favorecer derivaciones prácticas, contenidos y acciones que promuevan el desarrollo.

Las prácticas pedagógicas deben evitar la condición de excluidos -outsiders- de un grupo en comparación con otro. Es necesario que no haya distinción entre normales y anormales, competentes e incompetentes, cuando un alumno es elegible para la Atención Educativa Especializada, para el ingreso en escuelas especiales o para cualquier otra posibilidad de acción educativa.

Hay que respetar las diferencias de cada alumno para que no se establezcan parámetros inalcanzables. Cada evaluación debe ser pensada como única y respetar las potencialidades de cada individuo. Milanesi (2012), en una investigación en la cual estudiaba la realidad de profesores brasileños, apunta que la evaluación del rendimiento académico les angustia grandemente y resalta que el hecho de que, aun siendo indicados en el censo escolar, los alumnos con discapacidad, trastornos generales del desarrollo y altas habilidades/superdotación hacen las mismas evaluaciones que los otros. Los órganos de la educación nacional brasileña exigen que, para atender a esos alumnos, se adecuen los currículos, pero, a la hora de evaluar, la prueba no sufre ninguna adecuación.

Resulta muy difícil legislar los procesos de integración escolar; es que hay aspectos que el derecho natural no puede alcanzar. Sobre todas las cosas puede existir una legislación, una normativa, que regule las acciones de los actores psicoeducativos; sin embargo, nadie puede legislar los afectos. Así, cuando los maestros y/o psicólogos, psicopedagogos y todos los agentes psicoeducativos que intervienen en un proceso de integración rechazan ese proceso, esa normativa - no el niño, el alumno con NEE, sino las estrategias- o no comprenden el modo de llevarlas adelante, o no acuerdan con el principio de una escuela para todos, entre miles de otros modos de rechazo, los procesos de integración fracasan. En esos casos, se termina afirmando: "El alumno 'no es integrable"'.

Hay que pensar en evaluaciones que tengan al niño como parámetro. Es necesario que él sea el punto de partida y el punto de llegada de todas las intervenciones propuestas. Hay que pensar en procesos evaluativos que entiendan cada niño como el límite a ser alcanzado y que comparen cada conquista a su punto inicial. Sus progresos serán siempre grandes y valiosos, si 


\section{pro.posıções}

http://dx.doi.org/10.1590/1980-6248-2017-0116

\section{$e$-ISSN 1980-6248}

comparados a las ausencias y vacíos del inicio. También resulta fundamental comprender "al niño" situado en el contexto escolar, en su condición de alumno. Hay restricciones que solo se manifiestan y se hacen visibles en la escuela, no en la vida cotidiana del sujeto, es decir, que atañen a su condición de "niño situado en la escuela: alumno". Ese dato marca la importancia de la creación de estrategias pedagógicas —independientemente del diagnóstico- y, de allí, de la trascendencia de la intervención del maestro dentro de la sala de clases. Tal vez la interdisciplina sea la solución para esas situaciones, de modo que se constituya un grupo de profesionales, cada cual desde su perspectiva, que aporte y debata alcances y límites de su saber, al servicio de la educación de todos.

Así, no volveremos a cometer el error de hacer caer el peso de la integración (éxito o fracaso) sobre las peculiaridades del alumno, lo que es una situación injusta y éticamente reprochable. Los procesos de integración deben considerarse un desafío institucional y, por sobre todo, necesitan tener un lugar visible en las políticas educativas, nacionales y regionales.

\section{Referencias}

Anache, A. A., Rovetto, S. S. M., \& Oliveira, R., A. (2014). Desafios da implantação do atendimento educacional especializado no ensino superior. Revista Educação Especial, 27(49), 299-312.

Anache, A. A., Silva, A. M., Bruno, M. M. G., \& Martins, M. F. A. (2015). Atendimento educacional especializado nas salas de recursos multifuncionais no estado de Mato Grosso do Sul. In E. G. Mendes, F. Cia, \& S. D’Affonseca (Orgs.), Inclusão escolar e a avaliação do público alvo da Educação Especial (Vol. 1). São Carlos, SP: Manzine \& Marquezine.

Baquero, R. (2009). Desarrollo psicológico y escolarización en los enfoques socio-culturales: nuevos sentidos de un viejo problema. Avances en Psicología Latinoamericana, 27(2), 263 280. 


\section{pro.posições}

\section{$e$-ISSN 1980-6248}

Brasil. (2008). Ministério da Educação. Secretaria de Educação Especial. Política nacional de educação especial na perspectiva da educação inclusiva. Brasília: MEC.

Brasil. (2010). Ministério da Educação. Nota Técnica MEC/CGPEE/GAB n. 15, de 02 de julho de 2010 (Assunto: Orientações sobre Atendimento Educacional Especializado na Rede Privada). Brasília, DF.

Castorina, J. A. (2007). Las condiciones sociales de la investigación y los modelos de explicación. In J. Castorina, D. Aisenson, N. Elichiry, A. Lenzi, \& S. Schlemenson (Coords.), Aprendizajes, sujetos y escenarios. Investigaciones y prácticas en Psicología Educacional. Buenos Aires: Novedades Educativas.

Elias, N. (1994). A sociedade dos individuos. Rio de Janeiro: Jorge Zahar.

Elias, N., \& Scotson, J. L. (2000). Os estabelecidos e os outsiders: sociologia das relações de poder a partir de uma pequena comunidade. Rio de Janeiro: Jorge Zahar.

Fernandes, T. L. G., \& Viana, T. V. (2009, maio/agosto). Alunos com necessidades educacionais especiais (NEE): avaliar para o desenvolvimento pleno de suas capacidades. Estudos em Avaliação Educacional, 20(43), 305-318.

Kaplan, C. V. (2008). Talentos, dones e inteligencias. El fracaso escolar no es un destino. Buenos Aires: Colihue.

Martins, M. F. A., \& Sipes, M. L. (2015). Formação de professores na escola inclusiva: possibilidades, limites e aproximações entre Brasil e Argentina. Teoria e Prática da Educação: História da formação docente: olhares latino-americanos, 18(1), 37-46.

Melo, H. C. B. (2014). A versão das professoras das salas de recursos multifuncionais do município de Dourados-MS: atuação no atendimento educacional especializado. Dissertação de Mestrado em Educação, Programa de Pós-Graduação em Educação, Universidade Federal da Grande Dourados, Dourados-MS.

Melo, H. C. B., \& Martins, M. F. A. (2013). A avaliação e encaminhamento dos alunos com necessidades educacionais especiais - NEEs para as salas de recursos multifuncionaisSRMs em Dourados/MS. In Anais do VII Congresso Brasileiro Multidisciplinar de Educação Especial e do VIII Encontro da Associação Brasileira de Pesquisadores em Educação Especial (Vol. 01, pp. 75- 89). Londrina: EDUEL. 


\section{pro.posıções}

http://dx.doi.org/10.1590/1980-6248-2017-0116

\section{$e$-ISSN 1980-6248}

Mendes, E. G. (2006). A radicalização do debate sobre inclusão escolar no Brasil. Revista Brasileira de Educaşão, 11, 387-405.

Mendes, E. G., Cia, F., \& D’Affonseca, S. (Orgs.). (2015). Inclusão escolar e a avaliação do público alvo da Educação Especial (Vol. 1). São Carlos, SP: Manzine \& Marquezine.

Milanesi, J. B. (2012). Organização e funcionamento das salas de recursos multifuncionais em um município paulista. Dissertação de Mestrado em Educação Especial, Programa de Pós-Graduação em Educação Especial, Universidade Federal de São Carlos, São Carlos.

Rivière, A. (2003). Desarrollo y educación. El papel de la educación en el "diseño" del desarrollo humano. In A. Rivière, Obras escogidas (Vol. III Metarrepresentación y semiosis), Madrid: Editorial Médica Panamericana.

Schneider, R., \& Ravasio, M. T. H. (Orgs.) (2014). Norbert Elias: contribuições para a inclusão do aluno com deficiência no Ensino Superior. INTERFACES: Educação e Sociedade, 01, 35-54.

Sipes, M. (2012). Concepciones de la inteligencia en la práctica psicopedagógica. Entre el conservadurismo y la herejía. La lucha por el significado. Dissertação de Mestrado, Facultad de Psicología, Universidad de Buenos Aires, Argentina.

Unesco. (1994). Declaração de Salamanca. Sobre os Princípios, Políticas e Práticas na Área das Necessidades Educativas Especiais. Espanha.

Valsiner, J. (2012). La dialéctica en el estudio del desarrollo. In J. Castorina, \& M. Carretero (Eds.), Desarrollo cognitivo y educación (Vol. 1). Buenos Aires: Paidós.

Veltrone, A. A., \& Mendes, E. G. (2011, janeiro/abril). Caracterização dos profissionais responsáveis pela identificação da deficiência intelectual em escolares. Revista de Educação Especial, 24(39), 61-76.

\section{Legislación}

Constituição da República Federativa do Brasil de 1988. (1988, 5 de outubro). Brasília: Presidência da República. Casa Civil. Subchefia para Assuntos Jurídicos. Diário Oficial da União, ano CXXVI, n. 191-A. 
Decreto BR n. 7.611 de 17 de novembro de 2011. Dispõe sobre a educação especial, o atendimento educacional especializado e dá outras providências. Presidência da República, Brasília Brasil. (2011). Congresso Nacional, DF, 2011.

Sometido a evaluación el 29 de agosto de 2016; aprobado para publicación el 29 de agosto de 2017. 